\title{
TROMBOEMBOLISMO PULMONAR AGUDO EN LA REPÚBLICA ARGENTINA: PROTOCOLO DEL REGISTRO CONAREC XX
}

\author{
ACUTE PULMONARYTHROMBOEMBOLISM IN THE ARGENTINE \\ REPUBLIC: CONAREC XX REGISTRY PROTOCOL
}

\author{
IGNACIO M. CIGALINI', CRISTHIAN E. SCATULARO', JUAN C. JÁUREGUI', MAICO I. BERNAL', JAVIER I. ORTEGO', JUAN M. ABOY', \\ DANIEL CORNEJO', SEBASTIÁN GARCÍA ZAMORA', EZEQUIEL J. ZAIDEL'
}

\begin{abstract}
RESUMEN
Introducción. El tromboembolismo de pulmón agudo (TEP) representa la tercera causa de mortalidad cardiovascular. Sin embargo, existen pocos datos de esta patología en nuestro país.

Objetivo. Describir las características basales, evolución y tratamiento implementado en pacientes internados por TEP agudo en Argentina.

Métodos. Estudio multicéntrico, prospectivo y observacional de pacientes con diagnóstico de TEP agudo internados en centros con residencia de Cardiología desde octubre de 2016 a noviembre de 2017 independientemente si este fuere causa de la internación o surgiera como complicación de hospitalización por otra causa. Se remitirán datos en forma online a través de un sitio seguro con contraseña individual para cada centro participante. Se analizarán variables sociodemográficas, clínicas, estudios complementarios y evolución intrahospitalaria. Se realizará auditoría cruzada al 20\% de los centros.

Conclusiones. El registro CONAREC XX aportará valiosa información para conocer la realidad del TEP agudo en nuestro país.
\end{abstract}

Palabras clave: registros médicos; embolia pulmonar; Argentina.

\section{ABSTRACT}

Introduction: Acute pulmonary thromboembolism (PTE) represents the third cause of cardiovascular mortality. However, there are few data on this pathology in our country.

Objective: To describe the baseline characteristics, evolution and treatment implemented in patients hospitalized for acute PTE in Argentina.

Methods: Multicenter, prospective and observational study of patients diagnosed with acute PTE admitted to centers with cardiology residency from October 2016 to November 2017, regardless of whether this was due to hospitalization or as a complication of hospitalization due to another cause. Data will be sent online through a secure site with an individual password for each participating center. Sociodemographic, clinical variables, complementary studies and in-hospital evolution will be analyzed. Cross audits will be carried out on $20 \%$ of the centers. Conclusions: The CONAREC XX registry will provide valuable information to know the reality of acute PTE in our country.

Keywords: medical records; pulmonary embolism; Argentina.

REVISTA CONAREC 2018;33(146):244-245 | DOI:10.32407/RCON/2018146/0244-0245

\section{INTRODUCCIÓN}

El tromboembolismo de pulmón (TEP) representa la tercera causa de mortalidad cardiovascular', siendo responsable de al menos 100.000 muertes anuales en Estados Unidos ${ }^{2}$ y 300.000 en Europa ${ }^{3}$. Su presentación clínica variable y frecuentemente inespecífica resulta muchas veces un desafío diagnóstico con el consecuente riesgo de infraestimar su real incidencia ${ }^{4}$ e incluso retrasar el inicio de tratamiento específico que resultará en un peor pronóstico. Esta situación determina que se trate de la principal causa de mortalidad intrahospitalaria prevenible 5 .

A pesar de un tratamiento correcto, una significativa proporción de sobrevivientes deberán afrontar consecuencias invalidantes como riesgo aumentado de recurrencia ${ }^{6}$ e hipertensión pulmonar crónica, convirtiendo al TEP en la tercera causa de morbilidad cardiovascular luego de la cardiopatía isquémica y el accidente cerebrovascular? Si bien hay numerosos registros internacionales que han colaborado en conocer factores de riesgo y su historia natura|8-11, existe escasa información en nuestro medio acerca del manejo de esta patología ${ }^{12}$. Al mismo tiempo, a pesar de los avances y el creciente interés en el TEP, continúan existiendo grandes "zonas grises" y controversias especialmen-

\footnotetext{
1. Investigadores registro CONAREC XX
}

$\triangle$ Correspondencia: Dr. Ignacio Manuel Cigalini. Arribeños 3230. 4to piso, departamento 10.C1429BKJ CABA, Rep. Argentina.imcigalini@gmail.com.

Los autores declaran no tener conflictos de intereses.

Recibido: 27/08/2018 | Aceptado: 10/09/2018 te en lo que respecta al tratamiento en su fase aguda, como ser la reperfusión en pacientes con riesgo intermedio-alto o el desarrollo de diferentes métodos de reperfusión endovascular. Asimismo, no contamos con información respecto del impacto generado por la introducción de los anticoagulantes directos orales (ACOD) en la práctica clínica habitual. Estos factores motivaron que desde el Consejo Argentino de Residentes de Cardiología (CONAREC) se inicie un nuevo registro nacional que ayude a comprender cuál es la realidad del TEP en nuestro país en el marco de un programa superador que incluyó actividades de capacitación y difusión para residentes de cardiología y la comunidad en general ${ }^{13}$. El objetivo de este trabajo es describir las características basales, evolución y tratamiento implementado en pacientes internados por TEP agudo en Argentina. Como objetivos secundarios planteamos evaluar terapias de reperfusión de acuerdo a la estratificación de riesgo, identificar predictores de mortalidad intrahospitalaria, validar herramientas clínicas disponibles para estimar riesgo de eventos, evaluar diferencias en la evaluación diagnóstica y terapéutica de acuerdo a servicio responsable y área de internación; y analizar el perfil de pacientes que reciben ACOD en comparación con ensayos clínicos.

\section{MATERIALES Y MÉTODOS}

Estudio observacional, multicéntrico, prospectivo, con seguimiento limitado a la internación, realizado entre octubre de 2016 y noviembre de 2017. Se incluyeron pacientes de 16 años o más, admitidos a unidad coronaria, unidad de terapia intensiva o sala de internación general de centros con residencia de Cardiología afiliados al CONAREC, por episodio de tromboembolis- 
mo pulmonar agudo confirmado independientemente si fuera la causa de internación o surgiera como complicación de la internación por otra causa. Los datos serán recolectados por un médico residente de Cardiología, delegado de cada centro ante CONAREC y subinvestigador del estudio. Se obtendrán a partir de la entrevista personal a cada paciente durante la internación y de datos constatados en la historia clínica. La carga de datos se realizará on-line a través de un electronic case report form (Figura 1) diseñado en la plataforma LimeSurvey ${ }^{\circledR}$ con acceso exclusivo y único para cada centro a partir de una clave individual, con transferencia automática e inmediata a una base central en la plataforma. Para estratificar el riesgo de los pacientes se utilizará la clasificación sugerida por la European Society of Cardiology ${ }^{14}$, el Pulmonary Embolism Severity Score (PESI) $)^{15,16}$ y el PESI simplificado ${ }^{17}$. Asimismo, se evaluarán los puntajes RIETE ${ }^{18}$ y HAS-BLED ${ }^{19}$ para determinar el riesgo de sangrado. El análisis de los puntajes de riesgo se calculará en forma independiente y ciega a partir de las variables que los componen por el investigador principal. Se consideró la clasificación del Bleeding Academic Research Consortium (BARC) ${ }^{20}$ para definir y clasificar los sangrados. El resto de las definiciones se describen en el material suplementario.

Se realizará auditoría cruzada al 20\% de los centros participantes por un comité independiente. Se cotejarán variables aleatorias de la información remitida con la de las historias clínicas de las instituciones.

\section{ANÁLISIS ESTADÍSTICO}

Las variables continuas se expresarán con media y desvío estándar o mediana y rangos intercuartilos, dependiendo de la distribución de las mismas. Las variables categóricas serán expresadas como números y porcentajes. Para las comparaciones entre grupos se utilizará el test de Student o el test de suma de rangos de Wilcoxon, según corresponda.
Las comparaciones entre proporciones se realizará mediante el test de Chi cuadrado o el test exacto de Fisher, dependiendo de la frecuencia de valores esperados. En todos los casos se asumirá un error alfa del 5\% para establecer la significación estadística. Para el análisis estadístico se emplearán los programas estadísticos Epi Info 7.2 y STATA 13.

\section{CONSIDERACIONES ÉTICAS}

Se remitirá protocolo y consentimiento informado al comité de ética de cada centro para su aprobación. Los mismos se encuentran desarrollados en el material suplementario. No se obtendrán datos filiatorios de los pacientes, preservando su identidad.

\section{AUTORÍA}

La publicación de los resultados y posteriores subanálisis se realizarán bajo la autoría del CONAREC y del Comité Organizador del Registro. A los centros participantes se les entregará una certificación institucional como participantes del Registro y a los investigadores y colaboradores de cada centro se les dará una certificación como coautores del Registro CONAREC XX. Para la utilización de los datos del registro global, los investigadores deberán enviar una presentación con los objetivos del trabajo al Comité Organizador del registro de acuerdo se describe en la página oficial (www.conarec.org). El Comité Organizador será el encargado de administrar el acceso a la base de datos generales, para proteger el valor de la información del registro.

\section{CONCLUSIÓN}

El Registro CONAREC XX aportará información valiosa para conocer la realidad de los pacientes con TEP agudo atendidos en centros con residencias de cardiología de nuestro país.

\section{BIBLIOGRAFÍA}

1. Heit J. The epidemiology of venous thromboembolism in the community. Arterioscler Thromb Vasc Biol 2008:28(3):370-372

2. Horlander KT, Mannino DM, Leeper KV. Pulmonary embolism mortality in the United States, 1979-1998: an analysis using multiple-cause mortality data. Arch Intern Med 2003:163(14):1711-7.

3. Arya R. Venous Thromboembolism Prevention. A Patient Safety Priority. London: Department of Health; 2009 .

4. Cohen A, Agnelli G, Anderson F, Arcelus J, Bergavist D, Brecht J, et al. Venous thromboembolism (VTE) in Europe. The number of VTE events and associated morbidity and mortality. Thromb Haemost 2007;98(4):756-64.

5. Galson SK. The Surgeon General's Call to Action to Prevent Deep Vein Thrombosis and Pulmonary Embolism. U.S. Department of Health and Human Services; 2008.

6. Eichinger S, Weltermann A, Minar E, Stain M, Schounauer V, Scheider B, et al. Symptomatic pulmonary embolism and the risk of recurrent venous thromboembolism. Arch Intern Med 2004;1 (164):92-96.

7. Consenso de Enfermedad Tromboembólica. Consenso Argentino SAC. Rev Argent Cardiol 2009:77:411-28

8. Pollack C, Schreiber D, Goldhaber S, Slattery D, Fanikos J, O'Neil B, et al. Clinical characteristics, management, and outcomes of patients diagnosed with acute pulmonary embolism in the emergency department: initial report of EMPEROR (Multicenter Emergency Medicine Pulmonary Embolism in the Real World Registry). J Am Coll Cardiol 2011;57(6):700-706.

9. Laporte S, Mismetti P, De'cousus H, Uresandi F, Otero R, Lobo J, et al. Clinical predictors for fatal pulmonary embolism in 15,520 patients with venous thromboembolism: findings from the Registro Informatizado de la Enfermedad Tromboembólica venosa (RIETE) Registry. Circulation 2008:117(13):1711-1716.

10. Goldhaber S, Visani L, De Rosa M. Acute pulmonary embolism: clinical outcomes in the International Cooperative Pulmonary Embolism Registry (ICOPER). Lancet 1999;117(13):1386-1389.
11. Kasper W, Konstantinides S, Geibel A. Management strategies and determinants of outcome in acute major pulmonary embolism: results of a multicenter registry. J Am Coll Cardiol 1997;30:1165-1171.

12. Castillo Costa Y, Gómez Santa María H, Mauro V, Blanco Cerdeira P, Thierer J, Higa C. Registro Argentino de Enfermedad Tromboembólica Venosa. Rev Argent Cardiol 2013;81:251-257.

13. Scatularo CE. Tratamiento del tromboembolismo pulmonar agudo: controversias actuales. Rev Conarec 2017:(138):30-39.

14. Kostantinides SV, Torbicki A, Agnelli G, Danchin N, Fitzmaurice D, Galie N, et al. 2014 ESC Guidelines on the diagnosis and management of acute pulmonary embolism. Eur Heart J 2014;35(43):3033-3080.

15. Aujesky D, Obrosky D, Ra S, Auble T, Perrier A, Cornuz J, et al. Derivation and validation of a prognostic model for pulmonary embolism. Am J Respir Crit Care Med 2005;172(8):1041-6.

16. Donzé J, Le Gal G, Fine M, Roy P, Sanchez O, Verschuren F et al. Prospective validation of the Pulmonary Embolism Severity Index. A clinical prognostic model for pulmonary embolism. Thromb Haemost 2008;100(5):943-8.

17. Jiménez D, Aujesky D, Moores L, Gómez V, Lobo J, Uresandi F, et al. Simplification of the pulmonary embolism severity index for prognostication in patients with acute symptomatic pulmonary embolism. Arch Intern Med 2010;170(9):1383-9.

18. Ruíz-Giménez N, Suárez C, González R, Nieto J, Todolí J, Samperiz A, et al. Predictive variables for major bleeding events in patients presenting with documented acute venous thromboembolism. Findings from the RIETERegistry. Thromb Haemost 2008;100(1):26-31.

19. Kooiman J, van Hagen N, I lesias del Sol A, Planken E, Lip G, van der Meer F, et al. The HAS-BLED Score Identifies Patients with Acute Venous Thromboembolism at High Risk of Major Bleeding Complications during the First Six Months of Anticoagulant Treatment. PLOS ONE 2015:10(4):E0122520.

20. Mehran R, Rao S, Bhatt D, Gibson C, Caixeta A, Eikelboom J, et al. Standardized Bleeding Definitions for Cardiovascular Clinical Trials. A Consensus Report From the Bleeding Academic Research Consortium. Circulation 2011;123(23):2736-2747. 